\title{
Multiscale Modeling of the Deformation of Advanced Ferritic Steels for Generation IV Nuclear Energy
}

\section{NUCLEAR ENERGY RESEARCH INITIATIVE}

PI: Nasr M. Ghoniem, University of California, Los Angeles (UCLA)

Co-PI: Nick Kioussis, California State University, Northridge (CSUN)

Project Number: DE-FC07-06ID14748

Project Performance Period: 3/13/2006 - 3/12/2009

April 18, 2009 


\section{Table of Contents}

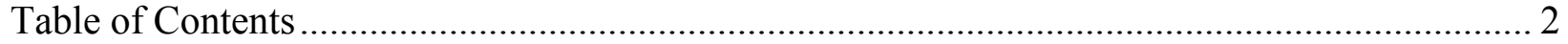

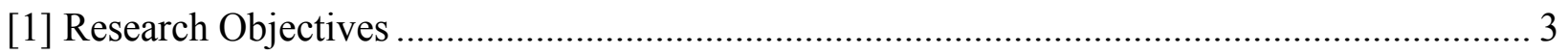

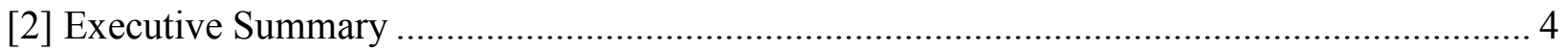

[2.1] Ab Initio Modeling of Dislocation-Precipitate Interaction ......................................... 4

[2.2] Core Structure of Self-Interstitial Clusters in Copper \& Iron ....................................... 5

[2.3] Dislocation Interaction with SIA Clusters \& Radiation Hardening ................................. 5

[2.4] Crystal Plasticity Modeling of Localized Deformation .............................................. 6

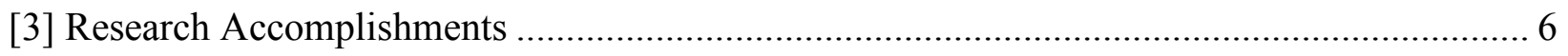

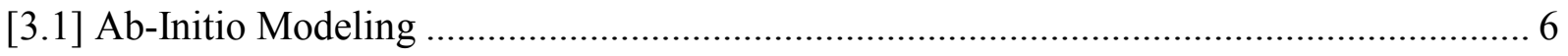

DEVELOPMENT OF A SEQUENTIAL MULTISCALE APPROACH - APPLICATION

TO Cu AND Cr NANOPRECIPITATES IN $\alpha$-IRON ............................................... 6

DEVELOPMENT OF A NOVEL CONCURRENT MULTISCALE APPROACH APPLICATION TO BCC METALS AND THE EFFECT OF CHEMISTRY ................... 9

ATOMISTIC SIMULATIONS OF Cu PRECIPITATES IN $\alpha$-Fe ................................. 12

INTERACTION OF A DISOCATION IN $\alpha$-IRON WITH AN Y2O3 PRECIPITATE..... 15

[3.2] Atomistic Modeling of the core structure of self-interstitial atoms in copper \& iron...... 16

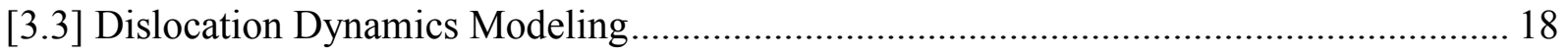

[3.4.] Constitutive \& Crystal Plasticity Modeling ............................................................ 22

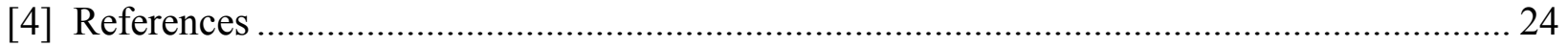




\section{[1] Research Objectives}

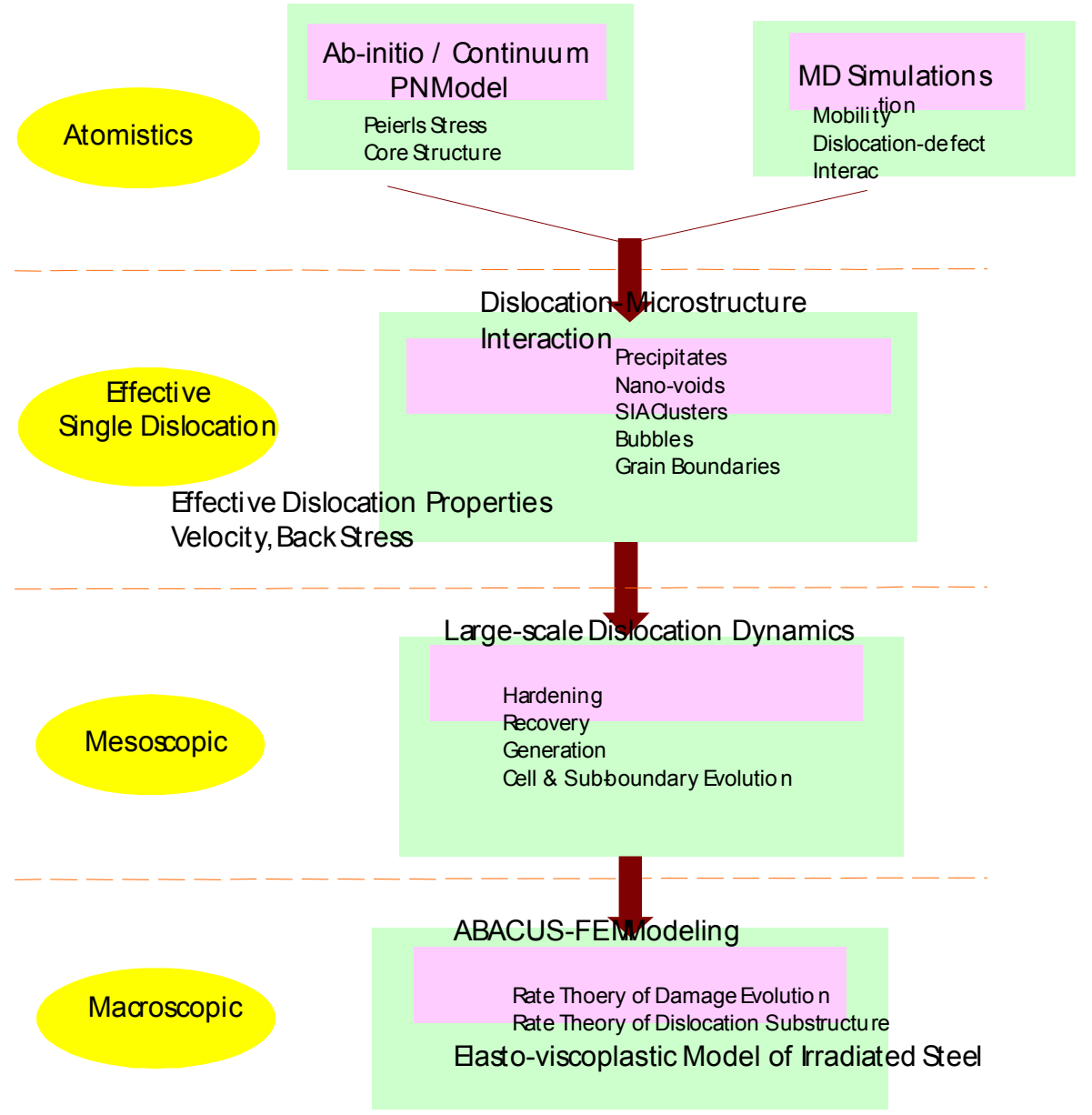

Figure (1): Overall structure of the Multi-scale Modeling Approach

The objective of this project is to use the multi-scale modeling of materials (MMM) approach to develop an improved understanding of the effects of neutron irradiation on the mechanical properties of high-temperature structural materials that are being developed or proposed for Gen IV applications. In particular, the research focuses on advanced ferritic/ martensitic steels to enable operation up to $650-700^{\circ} \mathrm{C}$, compared to the current $550^{\circ} \mathrm{C}$ limit on high-temperature steels. We develop here a multiscale modeling approach for in-reactor deformation (i.e. rateindependent plasticity, creep, and creep-fatigue) of advanced ferritic/ martensitic Gen-IV 
structural materials. Because empirical potentials are not well-developed for alloys and cannot easily adapt to local changes in chemistry due to impurities, we perform electronic structure calculations for basic inter-planar slip and cleavage in ferritic/ martensitic steels to describe energetics of twinning, dislocation slip, and inter-planar cleavage of the steels, accounting for local chemistry. We also complement these ab-initio calculations with traditional Molecular Dynamics (MD) simulations of dislocation-defect interactions. Through our recent generalization of the Peierls-Nabarro (PN) model, we simulate the dislocation core and twin properties with a hybrid ab-initio/ continuum 3-D model. One particular area of emphasis will be on dislocation interaction with oxide and carbide precipitates, which control the ductility and high-temperature strength of conventional and Oxide-Dispersion-Hardened (ODS) steels. Presently, there are no reliable methods at the atomistic scale, and our approach will solve this critical interaction problem and pass on the information to the micron length scale of a single dislocation. We then determine the in-reactor properties of single dislocations moving in radiation-damaged steels by simulating dislocation-defect interaction as a function of the neutron dose. Our method of Parametric Dislocation Dynamics (PDD) is extended to determine dislocation interaction with nano-voids, precipitates and Self Interstitial Clusters (SIA's), accounting for the first time for the flow of vacancies to dislocation cores during irradiation (i.e. dislocation climb). The effective properties of dislocations in radiation-damaged steels (e.g. stress-velocity relationship and back stress) are then be passed on to 3-D PDD simulations of the dislocation microstructure evolution under neutron irradiation. We will determine from these simulations rates of dislocation reactions (i.e. generation, recovery, hardening, and interactions with dislocation cells and subboundaries). These parameters will be used in a comprehensive Rate Theory model of radiation damage and in-reactor deformation developed earlier by the P.I. Extension of this model will focus on rate-independent (in-reactor) plasticity, high-temperature creep and creep-fatigue. The developed materials models are being integrated within the Finite Element framework through the ABACUS commercial FEM code. Predictions will be made for in-reactor deformation of simplified geometry, with full microstructure information linked with the deformation field.

\section{[2] Executive Summary}

\section{[2.1] Ab Initio Modeling of Dislocation-Precipitate Interaction}

One of the primary reasons for hardening of Fe-based alloys is the presence of precipitates in the Fe matrix induced by neutron irradiation. Cu-precipitates interact with dislocations in $\square-\mathrm{Fe}$, changing the mobility and/or dislocation core structure, thus inducing embitterment of $\square-\mathrm{Fe}$. $\mathrm{Ab}$ initio simulations are necessary to reveal the underlying mechanism for the interaction between the $\mathrm{Cu}$-precipitates and the screw dislocation in $\square$-Fe. Our results indicate that the dislocation core cannot be localized at the precipitate-matrix interface, namely the dislocation core spontaneously returns to the center of the precipitate. In addition, we find for the first time that the precipitate size has a large effect on the dislocation core polarization, which may affect the mobility. Spherical $\mathrm{Cu}$ precipitates larger than $2.0 \mathrm{~nm}$ have a polarized dislocation core, whereas 
the core is un-polarized for precipitates smaller than $1.3 \mathrm{~nm}$, similar to the un-polarized core in $\alpha-\mathrm{Fe}$.

\section{[2.2] Core Structure of Self-Interstitial Clusters in Copper \& Iron}

We developed a new computational method to analyze the core structure of dislocations and selfinterstitial loops in bcc iron and fcc copper, combining quantum mechanical determination of crystal lattice slip resistance with continuum mechanics for the elastic field. The method was originally developed by Banerjee, Ghoniem \& Kioussis based on the Pierels-Nabarro (PN) model and input of atomistic information prepared by separate ab-initio calculations. The core structure of dislocations can also be simulated by the classical Molecular Dynamics (MD) method. However, the reliability of the classical MD simulation result strongly depends on the accuracy of the interatomic potential, which is either empirically or semi-empirically developed by fitting an equation to available experimental and ab-inito calculation results. To determine the atomic arrangements in the dislocation cores using the hybrid method, we developed a new method that enables the calculation of displacements of atoms based on the elasticity of the infinitesimal dislocations, and the construction of atomic arrangements of the dislocation core.

\section{[2.3] Dislocation Interaction with SIA Clusters \& Radiation Hardening}

Self Interstitial Atom (SIA) clusters have a significant influence on damage evolution and the mechanical properties of irradiated materials. Kinetic Monte Carlo (KMC) computer simulations are performed to determine the kinetics of SIA cluster "clouds" in the vicinity of edge dislocations. The simulations include the elastic interactions between SIA clusters and between clusters and dislocations. Results of KMC simulations that describe the formation of clouds of SIA clusters in the presence of internal elastic fields during neutron irradiation of bcc Fe and the corresponding evolution kinetics are presented, and the size and spatial distribution of SIA clusters in the cloud region are studied for a variety of neutron displacement damage dose levels. The main features of the investigations are: (1) determination of the kinetics and spatial extent of defect clouds near static dislocations; (2) assessment of the influence of localized patches of SIA clouds on the pinning-depinning motion of dislocations in irradiated materials; (3) demonstration of the conditions for the formation of self-organized SIA raft patterns as a result of the interaction between mobile dislocations and glissile SIA clusters; and (4) estimation of the radiation hardening effects of SIA clusters. A study of the collective dynamics of thousands of SIA clusters, driven in their motion by their own interactions and by their interactions with moving dislocations is conducted to demonstrate some of the conditions for the emergence of a self-organized pattern of SIA cluster rafts. It is also shown that the critical stress to unlock trapped dislocations from SIA cluster clouds is in reasonable agreement with experimental observations. 


\section{[2.4] Crystal Plasticity Modeling of Localized Deformation}

The objective of this research is to develop an understanding of the mechanical behavior and dislocation microstructure evolution of single and polycrystals, and to delineate the physical and mechanical origins of spatially-localized plastic deformation in irradiated materials. A rateindependent crystal plasticity model was developed to incorporate micromechanics, crystallinity and microstructure into a continuum description of finite strain plasticity. A comprehensive dislocation density model based on rate theory is employed to determine the strain hardening behavior within each plastic slip system for the fcc crystal structure. Finite strain effects and the kinematics of crystal plasticity are coupled with the dislocation-density based model via the hardening matrix in crystal plasticity. The developed material models are applied to study single and polycrystal deformation behavior of copper. Interfaces between the ABAQUS user's subroutine Umat and the ABAQUS main code are developed to allow further extension of the current method. Simulations carried out for polycrystals clearly illustrate the heterogeneous nature of plastic strain, and the corresponding spatial heterogeneity of the mobile dislocation density. The origins of the spatial heterogeneities are essentially geometric, as a result of constraints on grain rotation (finite strain effects), geometric softening due to plastic unloading of neighboring crystals.

\section{[3] Research Accomplishments}

\section{[3.1] Ab-Initio Modeling}

DEVELOPMENT OF A SEQUENTIAL MULTISCALE APPROACH - APPLICATION TO $\mathrm{Cu}$ AND Cr NANOPRECIPITATES IN $\alpha$-IRON

At low temperatures, plasticity in bcc Fe is governed by low mobility screw dislocations with Burgers vector $\mathbf{b}=a / 2<111>$. It is generally believed that the core structure of these dislocations in bcc metals is a controlling factor of their mobility. For the non-magnetic bcc tantalum (Ta) theoretical studies have led to two types of core structures: Asymmetric core and symmetric core [1]. On the other hand, the dislocation core structure of bcc Fe has not been studied to date, except by using empirical interatomic potentials which can not capture accurately the additional complication arising from the presence of magnetism [2]. Furthermore, the interaction between dislocations and solute atoms if of great interest since it determines one of the important strengthening mechanisms in ferritic steel alloys, the so-called solid solution hardening (SHH).

The $\alpha-\mathrm{Fe}-\mathrm{X}$ base alloys $(X=\mathrm{Cr}, \mathrm{Cu}, \mathrm{Ni}$, etc.) are ideal systems to study SSH due to the very low solubility of $X$ in $\mathrm{Fe}$ at low temperatures and the importance of solute precipitation on the irradiation hardening and embrittlement of low alloy reactor pressure vessel steels. Previously, studies based on empirical interatomic potentials were used to study precipitate interactions in $\mathrm{Fe}-\mathrm{Cu}$ and have shown that about $3 \mathrm{~nm} \mathrm{Cu}$ precipitates strengthen $\alpha-\mathrm{Fe}$. However, the origin of the electronic structure responsible for the SSS or SSH in $\alpha$-Fe remains an unexplored area thus far because of the inadequacy of empirical potentials. The purpose of this work was to carry out a theoretical study of the effect of $\mathrm{Cu}$ or $\mathrm{Cr}$ solutes and solute clusters on the dislocation core 
properties of the $a / 2<111>$ screw dislocation in $\alpha$-Fe. We employ an ab initio based approach whose results are corroborated by complementary atomistic simulations using empirical interatomic potentials. The calculations reveal that $\mathrm{Cu}$ nanoprecipitates induce a dramatic change in the core structure from the nonpolarized in pure Fe to the polarized. In sharp contrast, it was shown that $\mathrm{Cr}$ precipitates have small effect on the core polarization and increase the Peierls stress. The underlying atomistic mechanism responsible for these uniquely electronic structure effects was elucidated.

$A b$ initio calculations of dislocations and dislocation-solute interactions, even though most accurate, are computationally expensive. As a result, most atomistic simulations are based on empirical descriptions of atomic interactions. However, such approaches depend critically on the interatomic potentials which are determined by fitting to experimental or ab initio data, and hence they are limited to describing accurately the effects of alloying. The hybrid ab initio based approach of the atomic-row (AR) model of Suzuki for a screw dislocation in bcc metals offers a plausible alternative. It serves as a link between $a b$ initio and atomistic approaches and represents a compromise between computationally efficiency and accuracy. The inter-row potential (IRP), derived from ab initio calculations, allows the treatment of solutes in the core, while the dislocation core structure is determined by relaxing the ARs using the IRP, similar to atomistic simulations employing interatomic interactions. The dislocation core of a screw dislocation can be described by the displacement of the atomic rows along the dislocation line. The interatomic row potential, is assumed to be a function only of the relative atomic positions, $\mathrm{u}$, and composed of the first and second terms in the Fourier expansion [3]

$\Phi(u)=\frac{\Phi_{0}}{(1-4 \alpha)}\left(\cos \left(\frac{2 \pi u}{b}\right)-\alpha \cos \left(\frac{4 \pi u}{b}\right)+\frac{1}{2}(1-\alpha)\right)$

where $\mathrm{b}$ is the burgers vector, and the parameters $\Phi_{0}$ and $\alpha$ is determined by the magnitude of the potential at $\mathrm{u}=0$ and $\alpha=0$. The parameter $\alpha \square$ describes the deviation of $\Phi(u)$ from a sinusoidal form. The average variation under the atomic row shift along <111>, $\Delta E_{\text {row }}=3[\Phi(u-1 / 3)+\Phi(u+1 / 3)]$, may be calculated using high accuracy first-principles electronic structure methods and the parameters $\Phi_{0}$ and $\alpha$ may be found from Eqs. (1) and (2). We have carried out total-energy calculations using the VASP code with the generalized gradient approximation (GGA) for the exchange-correlation potential. We have calculated the atomic row displacement energies for a shift of a single atomic row along the $<111>$ direction using supercells with vectors $\mathbf{a}_{1}=(2,-2 \sqrt{2}, 0) a, \mathbf{a}_{2}=(1, \sqrt{2}, 0) a$, and $\mathbf{a}_{3}=(0,0,4 \sqrt{2}) \mathrm{a}$ (a is the lattice constant) and consisting of 5 layers and 8 atoms. 


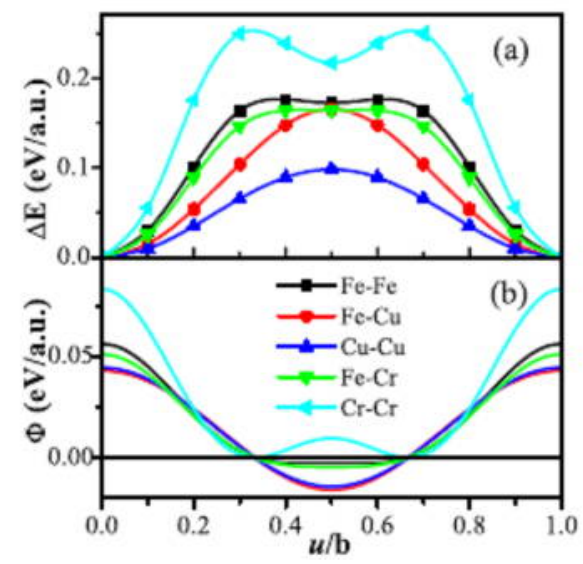

Figure 2: (a) Energy change per unit length, $\Delta E^{(F e-X)}$ and $\Delta E^{(X-X)}$ for $X=F e, C u$, and $C r$, versus the normalized $A R$ displacement $u / b$ along $<1111>$. (b) Corresponding variation of IRP, $\Phi^{F e-X}$ and $\Phi^{X-X}$.

Fig. 2(a) shows $\Delta E^{(F e-X)}$ and $\Delta E^{(X-X)}$ versus $u / b$ for $X$ $=\mathrm{Fe}, \mathrm{Cu}$, and $\mathrm{Cr}$. The corresponding IRP, $\Phi^{F e-X}$ and $\Phi^{X-X}$, versus $u / b$ are shown in Fig. 1(b). For $\mathrm{Fe}-\mathrm{Fe}, \mathrm{Fe}-\mathrm{Cr}$, and $\mathrm{Cr}-\mathrm{Cr}, \Delta E$ exhibits two well-pronounced maxima at $u=b / 3$ and $u=2 b / 3$ associated with both the bcc structure and the unfilled $3 d$ band for Fe and the solute atom. On the other hand, $\mathrm{Cu}$ solutes reduce dramatically the atomic-row shear energies for the $\mathrm{Fe}-\mathrm{Cu}$ and $\mathrm{Cu}$ $\mathrm{Cu}$ rows. Moreover, there is a change of the shape of $\Delta E$, which displays a single maximum at $u=b / 2$ for both $\mathrm{Fe}-\mathrm{Cu}$ and $\mathrm{Cu}-\mathrm{Cu}$. These results clearly demonstrate that $\mathrm{Cu}$ solutes act as lubricants and facilitate the shear process between the $\mathrm{Fe}-\mathrm{Cu}$ and $\mathrm{Cu}-\mathrm{Cu}$ rows. Analysis of the density of states and charge density indicate that the $\mathrm{NN}$ inter-row interaction for $\mathrm{Fe}-\mathrm{Fe}, \mathrm{Fe}-\mathrm{Cr}$, and $\mathrm{Cr}-\mathrm{Cr}$ is dominated by $t_{2 g}-t_{2 g}$ hybridization at the Fermi energy.

In Fig. 3(a), we show the relaxed dislocation core structure for pure Fe employing the differential displacement (DD) maps for the screw component using the ab initio based IRP approach. The arrows indicate the relative $<111>$ displacement of neighboring atoms produced by the dislocation. The length of the arrow is proportional to the magnitude of the displacement difference and the direction of the arrow indicates the sign of the displacement difference. When the arrow touches the centers of the two atoms, their relative displacement is $b / 3$. The orientation of all the (110) and (112) planes belonging to the $<111>$ zone is shown in Fig. 3(b). The dislocation core for pure Fe is symmetric (nondegenerate) and spreads symmetrically on the six planes, in agreement with recent fully $a b$ initio [4] and atomistic [5] calculations. The core structure for a small $\mathrm{Cu}$ cluster composed of three rows along the dislocation core, as shown in Fig. 3(b), changes dramatically from symmetric to nonsymmetric (degenerate), where the dislocation core spreads primarily into three (110) planes. Thus, the $2.5 \AA$ size $\mathrm{Cu}$ cluster reduces the core nonplanarity and renders it to more planar. In sharp contrast, the three atomic row $\mathrm{Cr}$ precipitate in Fig. 2(c) has a very small effect on the core structure, i.e., the core remains isotropic as in the case of pure Fe. 


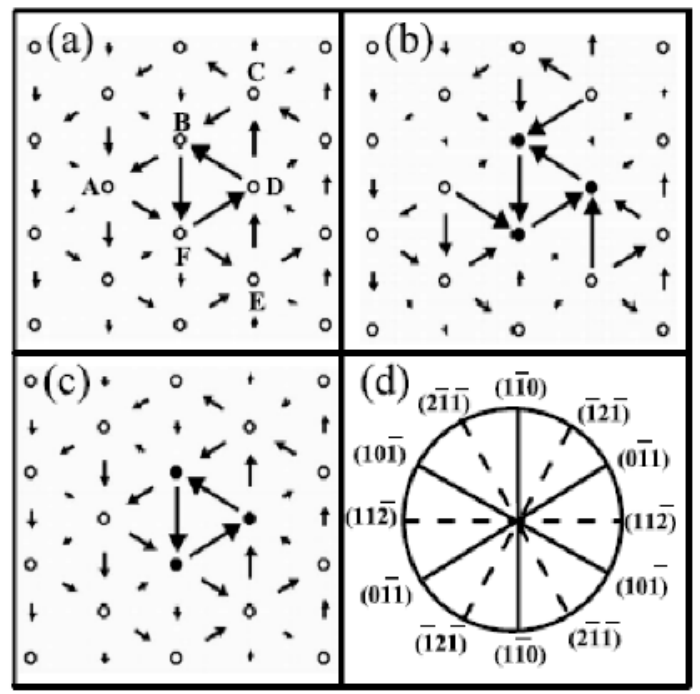

Figure 3 DD map of the core for a $a / 2<111>$ screw dislocation in (a) pure $\mathrm{Fe}$, (b) $\mathrm{Fe}-\mathrm{Cu}$, and (c) $\mathrm{Fe}-\mathrm{Cr}$, calculated from the $a b$ initio based IRP approach. The hollow circles and solid circles represent $\mathrm{Fe}$ and impurity atoms, respectively. (d) [111] stereographic projection of all $\{110\}$ and $\{112\}$ planes belonging to the [111] zone.

DEVELOPMENT OF A NOVEL CONCURRENT MULTISCALE APPROACH APPLICATION TO BCC METALS AND THE EFFECT OF CHEMISTRY

Solute atoms are ubiquitous in metals and play a key role in altering their mechanical properties e.g., strength and ductility. Experimental studies during the past several decades indicate that solutes can give rise to both solid-solution hardening ( $\mathrm{SSH}$ ) and solid-solution softening (SSS) [6].The origin of SSH/SSS is due to the interaction between dislocations and solutes and/or precipitates in materials. In most situations, the dislocation behavior is considerably different in the realistically dirty materials, where dislocation mobility can vary by several orders of magnitude. This significant influence induced by small amounts of solutes is of great practical importance to, for example, the bcc refractory metals ( $\mathrm{Nb}, \mathrm{W}$, Ta, and $\mathrm{Mo}$ ). These systems exhibit unique mechanical properties that make them attractive for structural applications at elevated temperatures. An inherent drawback limiting the use of these materials as structural components is their reduced low-temperature toughness, which in turn increases the propensity toward fracture. Thus, the challenge in design of advanced alloys is to combine strengthening and toughening phases with a better balance of properties. Continuum elasticity theory has provided considerable insight of SSS/SSH in terms of the size and elastic constants between the solute and host atoms. The correlation between the hardening rate and number of conduction electrons of transition-metal solutes, however, indicates a nonlinear chemical origin of the dislocation-solute interaction. [7, 8] In their pioneering work, Trinkle and Woodward using the first-principles Greens function boundary condition (FPGFBC) method, demonstrated the transition-metal solutes can have a large effect on the dislocation core and hence the mobility [9]. Nevertheless, understanding the physics of interactions of dislocations with nanoclusters remains a challenging problem.

We have developed a concurrent multiscale approach, which opens the door to studying the important problem of chemistry effect on the mechanical properties of metals. This approach 
treats correctly the long-range elastic field of the dislocation and describes the solute-host atomic interaction in the core region accurately. We have applied this approach to the Ta-W alloys, two prototype bcc metals, because (1) experiments have shown the dual nature of $\mathrm{W}$ : the addition of 2.5-10.0 wt.\% W in Ta increases the strength while it enhances substantially the ductility at high strain-rates; and (2) the high solubility of $\mathrm{W}$ in Ta allows a wide range of local environments. The results demonstrate the multi-nature of $\mathrm{W}$ solutes. Depending on the solute local environment random solid solution or nanoclusters of different geometry, the mobility may exhibit a wide spectrum of unusual behavior: SSS, SSH, or even a spontaneous dislocation glide in the absence of external stress. Finally, W solutes can serve as obstacles to dislocation motion and activate new slip planes.

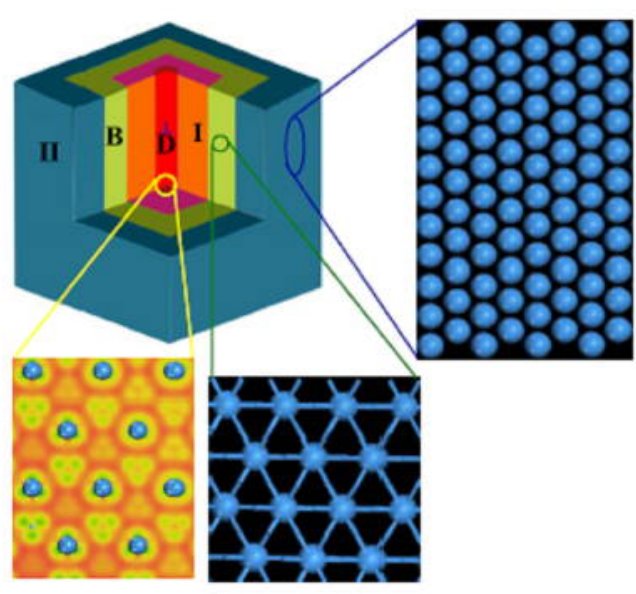

Figure 4 Schematic view of the partitioned multiscale system: Region I (red/gray) contains the core of the screw dislocation (marked by D); Region II (blue/dark gray) consists of atoms treated with EAM; and the boundary $(\boldsymbol{B})$ region (green/light gray) consists of atoms on the boundary between regions $I$ and II. Periodic boundary conditions are used along the (111) dislocation line.

The concurrent multiscale approach is based on that proposed by Choly et al. [10] The system, shown in Fig. 4, is divided into: region I, which includes the dislocation core and the solutes, is treated within the framework of density functional theory (DFT); region II which refers to the rest of the system is treated with the embedded atom method (EAM) or Finnis-Sinclair (FS) potentials; and the boundary (B) region introduced to reduce the coupling errors between regions I and II, respectively.

The total energy of the combined systems can be written in the form, $\mathrm{E}[\mathrm{I}+\mathrm{II}]=\mathrm{E}_{\mathrm{DFT}}[\mathrm{I}]+\mathrm{E}_{\mathrm{EAM}}[\mathrm{II}]+\mathrm{E}_{\text {int }}[\mathrm{I}, \mathrm{II}]$. One can show that the total energy of the coupled system can be written in the form, $\mathrm{E}[\mathrm{I}+\mathrm{II}]=\mathrm{E}_{\mathrm{EAM}}[\mathrm{I}+\mathrm{II}]-\mathrm{E}_{\mathrm{EAM}}\left[[\mathrm{I}]+\mathrm{E}_{\mathrm{DFT}}[\mathrm{I}]\right.$, where $\mathrm{E}_{\mathrm{EAM}}$ and $\mathrm{E}_{\mathrm{DFT}}$ are the energy of region I in the absence of II obtained by DFT and EAM calculations, respectively.

In Fig. 5 we show the relaxed dislocation core structure in pure Ta under zero stress (left panel) and $1.8 \mathrm{GPa}$ (right panel), respectively, using the differential displacement (DD) maps. The arrows indicate the relative (111) displacement of neighboring atoms of the dislocation. The length (direction) of the arrow denotes the magnitude (sign) of the displacement difference. 
When the arrow touches the centers of the two atoms, their relative displacement is $b / 3$. The dislocation core for pure Ta is nondegenerate and spreads symmetrically on the six planes. Upon increasing $\sigma_{y z}$ on the (101) planes, the dislocation center moves to the next Peierls valley at the critical stress $\sigma_{P}$ of $1.8 \mathrm{GPa}$. Comparison of $\sigma_{P}$ and the core structure in Table I employing different approaches demonstrates that the concurrent multiscale approach is in excellent agreement with the results of the FP-GFBC method.

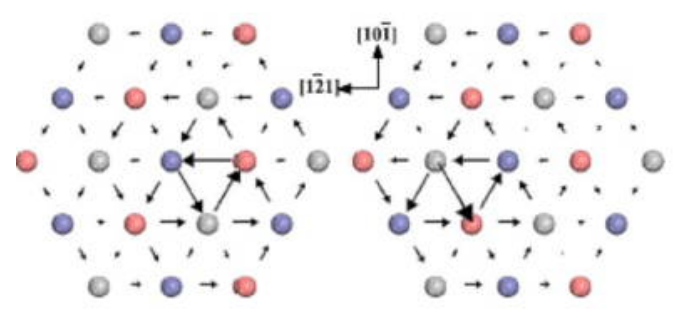

Figure 5 DD map of the core of the screw dislocation in Ta under (a) zero stress (left panel) and (b) $1.8 \mathrm{GPa}$ (right panel). Circles of different color (brightness) represent atoms on three successive (111) planes.

TABLE I. Peierls stress $\left(\sigma_{P}\right)$ and core structure for a screw dislocation in Ta using the present multiscale approach, the FPGFBC method, the EAM, and the modified generalized pseudopotential theory (MGPT).

$\underline{\sigma}_{\mathbf{P}}(\mathbf{G P a})$

present work

FP-GFBC [9]

EAM [11]
1.8

1.8

1.8

\section{Core Structure}

Nondegenerate

Nondegenerate

Degenerate

MGPT [12] $\quad 0.6 \quad$ Nondegenerate

In order to study the effect of local environment of the solute on the mobility of the screw dislocation, we have considered the cases of (i) dilute random solid solutions where the dislocation line is affected only by isolated solute atoms (ISA) shown in Fig. 6(a), and (ii) small clusters of solute atoms of hexagonal shape (CSA-H) shown in Fig. 6(b) or of triangular shape (CSA-T) shown in Fig. 6(c). In all cases the $\mathrm{W}$ concentration is about 6 at $\%$ and the $\mathrm{W}$ atoms are denoted with black circles. The upward triangle, downward triangle, square, and solid dot denote the position of the dislocation center under $0.0,1.0,1.5$, and $2.0 \mathrm{GPa}$, respectively. In the absence of stress the core center is at point in panel (d) for the ISA and the CSA-T cases, while it is unstable [dashed cross in panel (b)] for the CSA-H case. Interestingly, in the latter case the dislocation center is repelled by the CSA-H and spontaneously moves to the position of the 
upward triangle under the precipitate-induced chemical stress. Under 1.0 GPa, both in the ISA and CSA-H cases the dislocation prefers to bypass the $\mathrm{W}$ sites and moves on the (011) plane. Upon increasing the stress to $1.5 \mathrm{GPa}$, the behavior changes: the dislocation in ISA continues to move, while it is pinned in CSA-H. The dislocation in CSA-H begins to move further under 1.8 $\mathrm{GPa}$, as in the case in pure Ta. In sharp contrast to the other two cases, the dislocation core is pinned at $\Gamma$ in the CSA-T case until the stress reaches $2.0 \mathrm{GPa}$, where the center moves on the (110) glide plane. Both ISA and CSA-H result in SSS, while CSA-T produces SSH. In all cases, $\mathrm{W}$ solutes have a small effect on the polarization, indicating the absence of correlation between the polarization and $\sigma_{P}$.

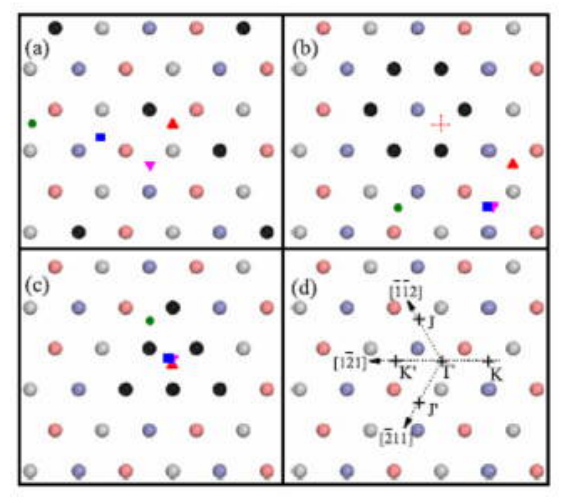

Figure 6 Screw dislocation slip paths under stress for three configurations of $W$ solutes denoted by black circles: (a) isolated solute atoms ISA and nanoclusters of (b) hexagonal (CSA-H) or (c) triangular (CSA-T) shape. The upward triangle, downward triangle, square, and solid dot denote the position of the core center under $0.0,1.0,1.5$, and $2.0 \mathrm{GPa}$, respectively. Panel (d) displays the special points and directions. $\Gamma$ indicates the initial position of the dislocation.

\section{ATOMISTIC SIMULATIONS OF Cu PRECIPITATES IN $\alpha$-Fe}

To predict, estimate and extend the operational life of RPV steels, one needs to understand the mechanism of irradiation-induced hardening. One of the main reasons for the hardening phenomenon is the nucleation and growth of $\mathrm{Cu}$ precipitates. Experimental observations have shown that ultrafine $\mathrm{Cu}$ precipitates with bcc structure serve as obstacles to impede dislocation motion in the Fe matrix. Lozano-Perez et al. found experimental evidence of the transformation of $\mathrm{Cu}$ precipitates (diameter $d \sim 4 \mathrm{~nm}$ ) from the bcc to the 9R phase [13]. This transformation is accompanied with the formation of Orowan loops. Recently, Nogiwa et al. showed that even smaller $\mathrm{Cu}$ precipitates which $d \sim 2 \mathrm{~nm}$ can curve and pin dislocations without Orowan loops [14]. In order to understand the difference between these observations, we have carried comprehensive atomistic simulations of the interaction between dislocations with $\mathrm{Cu}$ precipitates of various sizes. 


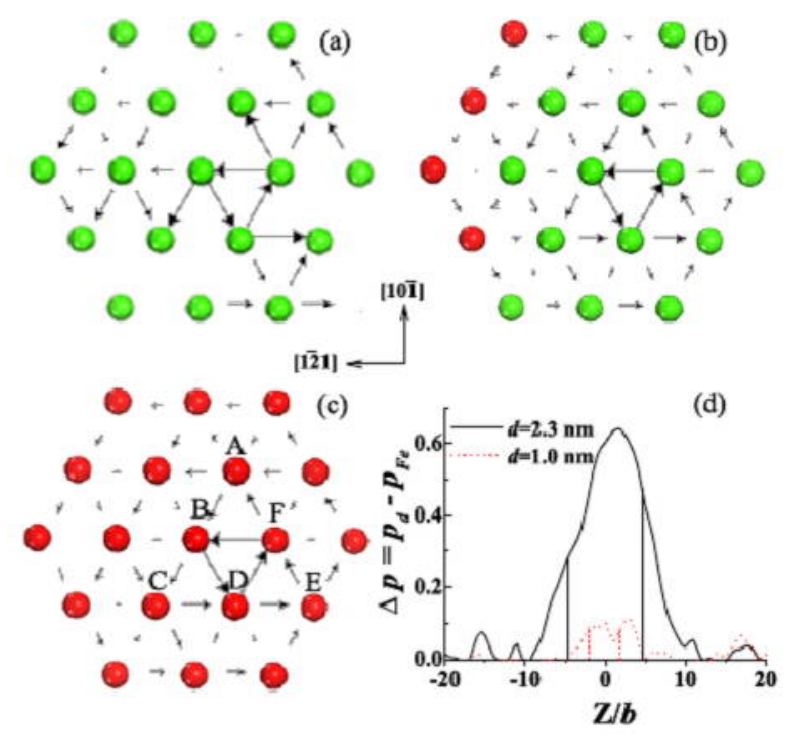

Figure 7 Dislocation core structure inside a Cu precipitate with (a) $d=2.3 \mathrm{~nm}$; (b) $d=1.0 \mathrm{~nm}$, and (c) $d=0.0 \mathrm{~nm}$ (Pure a-Fe). The red and green circles represent $\mathrm{Fe}$ and $\mathrm{Cu}$ atoms, respectively. (d) shows the change of dislocation core polarization versus atomic position (normalized to Burgers vector) along the dislocation line. The black solid and red dashed curves represent the $2.3 \mathrm{~nm}$ and $1.0 \mathrm{~nm} C u$ precipitates, respectively. The vertical dashed lines indicate the precipitate-matrix interface.

We have performed molecular dynamics (MD) simulation with system size of $12.2 \times 28.0 \times 19.9$ $\mathrm{nm}^{3}$. Three sizes of $\mathrm{Cu}$ precipitate with $d$ of $1.0 \mathrm{~nm}, 2.3 \mathrm{~nm}$ and $4.4 \mathrm{~nm}$ have been employed to study the size effect. A $\langle 111\rangle / 2$ screw dislocation is placed initially $7.0 \mathrm{~nm}$ away from the center of the precipitate. The shear stress is applied on the dislocation with strain ratee $=4 \times 10^{\square} / \mathrm{s}$. The results show that the $1.0 \mathrm{~nm} \mathrm{Cu}$ precipitate does not affect the motion of the dislocation, and hence does not contribute to the hardening effect. While the dislocation can penetrate into the $2.3 \mathrm{~nm} \mathrm{Cu}$ precipitate, initially becomes pinned and finally it curves as it moves out of the precipitate. We find that the bow-out angle $\theta=144^{\circ}$, in agreement with Nogiwa's observation [15]. Interestingly, we found that the $2.3 \mathrm{~nm}$ $\mathrm{Cu}$ precipitate changes the dislocation core polarization. When the dislocation moves out, it undergoes a core-structure transformation from a polarized to a non-polarized one, and the dislocation line curves in order to supply the extra stress to drive this transformation (Shown in Fig. 7). Thus, for the first time, our results reveal a novel polarized-induced strengthening mechanism of ultrafine $\mathrm{Cu}$ precipitates. 


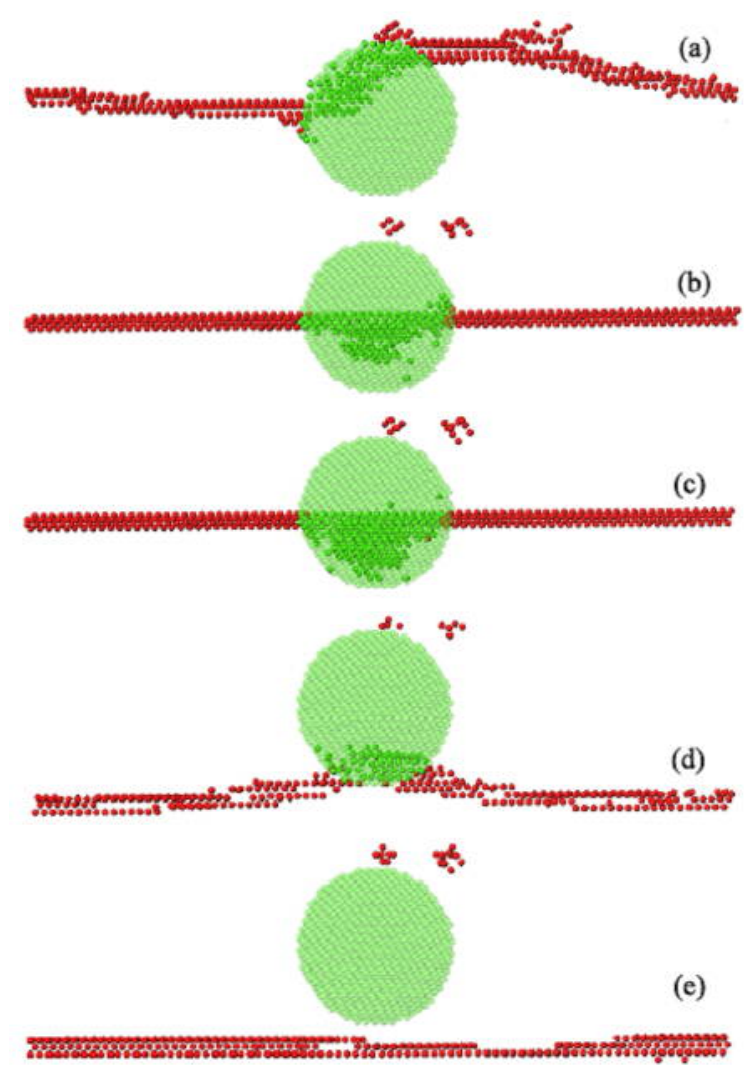

Figure 8 Snapshots of the dislocation core for the $4.4 \mathrm{~nm} C \mathrm{Cu}$ precipitate.

Finally, the $4.4 \mathrm{~nm} \mathrm{Cu}$ precipitate can dramatically impede the dislocation motion. As shown in Fig. 8, the dislocation stops at the center of the precipitate. With increasing external stress, an increasing number of $\mathrm{Cu}$ atoms become displaced from the perfect bcc sites. Consequently, the dislocation core spreads and expands in the precipitate. This observation is consistent with Lozano-Perez's result: the presence of the dislocation triggers the martensitic structural phase transformation, and the deformed precipitate impedes in turn the movement of dislocations. Thus, we find two different mechanisms that are responsible for the different experimental observations. The first, is a polarization-induced mechanism for $\mathrm{Cu}$ precipitates with $d \sim 2 \mathrm{~nm}$, while the second, is a deformation-induced mechanism for $\mathrm{Cu}$ precipitates with $d$ $>4 \mathrm{~nm}$. 


\section{INTERACTION OF A DISOCATION IN $\alpha$-IRON WITH AN Y2O3 PRECIPITATE}

Oxide dispersion strengthened (ODS) steels are promising candidates as structural materials for applications in fission and fusion reactors. $\mathrm{Y}_{2} \mathrm{O}_{3}$ particles dispersed in an iron matrix drastically improve the strength, without adverse effects on ductility. However, the interaction between a dislocation and the $\mathrm{Y}_{2} \mathrm{O}_{3}$ particle, considered to be the basic strengthening mechanism in ODS steels, remains unresolved. The objective of this work is to develop a sequential multiscale approach, which links dislocation dynamics with $a b$ initio input for the generalized stacking fault energy surface ( $\gamma$-surface), responsible for the lattice restoring force. This is an extension of the original Peierls-Nabarro model for interfaces. The Burgers vector of the screw dislocation is divided into fractional dislocations which are allowed to spread into the glide planes of the matrix, the precipitate, and the precipitate/matrix interface. The GSFES for these three sets of planes is calculated from $a b$ initio calculations. We take into account on an equal footing both the screw and edge components of the dislocation displacement field, which satisfies a number of boundary conditions. The approach takes into account both the elastic interaction and the misfit energy on an equal footing. We have developed the codes and we are currently testing them. As a first step, we have calculated the $\gamma$-surface of $\mathrm{Y}_{2} \mathrm{O}_{3}$ on the (001) plane along the [100] and [110] directions, shown in Fig. 9(a) and Fig. 9(b), respectively. We have also calculated the $\gamma$-surface of the $\mathrm{Y}_{2} \mathrm{O}_{3}-\alpha-\mathrm{Fe}$ interface, which will be employed to carry out the dislocation dynamics calculations to investigate the core structure, and compare with experimental observations [16,17].
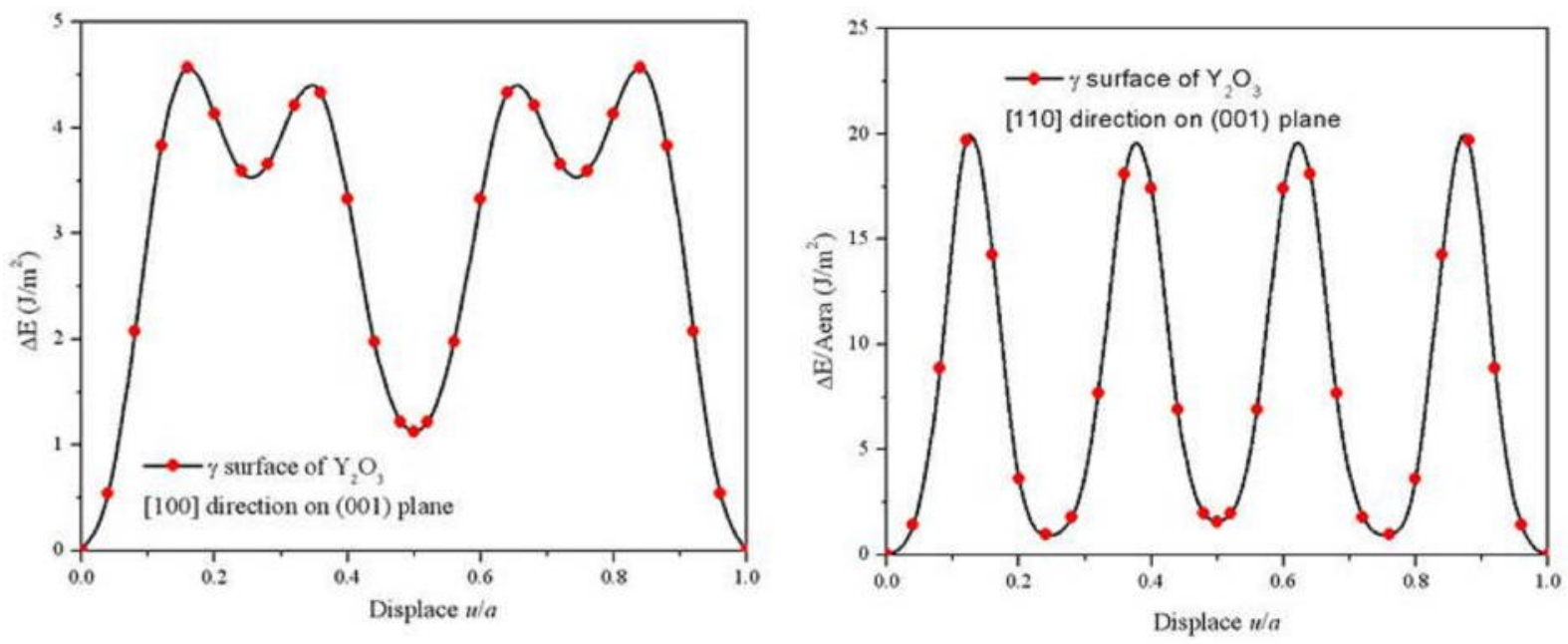

Figure $9 \gamma$-surface of $\mathrm{Y}_{2} \mathrm{O}_{3}$ on the (001) plane. Left panel is along the [100] direction and right panel is along [110] direction. 


\section{[3.2] Atomistic Modeling of the core structure of self-interstitial atoms in copper \& iron}

The dislocation core structure of Self-Interstitial Atom (SIA) clusters in bcc iron and fcc copper is determined using the hybrid ab initio -continuum method of Banerjee et al.1. To reduce reliance on empirical potentials, and to facilitate predictions of the e ${ }^{\circledR e c t s}$ of local chemistry and stress on the structure of defects, we present here a hybrid extension of the Pierels-Nabarro (PN) continuum model, with lattice resistance to slip determined separately from ab initio calculations. A method is developed to reconstruct the atomic arrangement and geometry of SIA clusters from the hybrid model. The results are shown to compare well with Molecular Dynamics (MD) simulations. In iron, the core structure does not show dependence on the size of the selfinterstitial cluster, and is nearly identical to that of a straight edge dislocation. However, the core structure of SIA clusters in $\mathrm{Cu}$ is shown to depend strongly on the cluster size. Small SIA clusters are found to have non-dissociated compact dislocation cores, with strong merging of Shockley partial dislocations and a relatively narrow stacking fault (SF) region. The compact nature of the SIA core in copper is attributed to the strong dependence of the self-energy on the cluster size. As the number of atoms in the SIA cluster increases, Shockley partial dislocations separate and the SF region widens, rendering the SIA core structure to that of an edge dislocation. The separation distance between the two partials widens as the cluster size increases, and tends to the value of a straight edge dislocation for cluster sizes above 400 atoms. The local stress is found to have a significant effect on atomic arrangements within SIA clusters in copper and the width of the stacking faults. For an SIA cluster containing 400 atoms, $1 \mathrm{GPa}$ shear stress delocalizes the cluster and expands the SF to $30 \mathrm{~b}$, while an opposite shear stress of $2 \mathrm{GPa}$ contracts the core to less than $5 \mathrm{~b}$, where $\mathrm{b}$ is the Burgers vector magnitude. The Displacement density distribution for the dislocation core of self-interstitial loops in iron is shown in Figure (10). The core structure of a straight edge dislocation is also plotted. Figure (11) shows the SIA dislocation core structure in copper. 


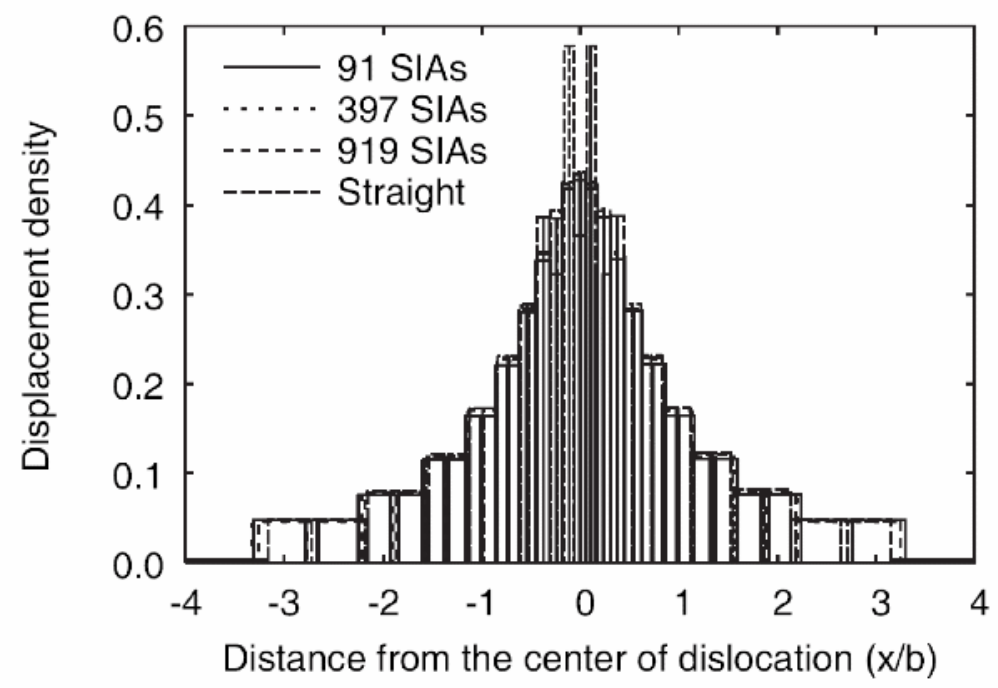

Figure (10)

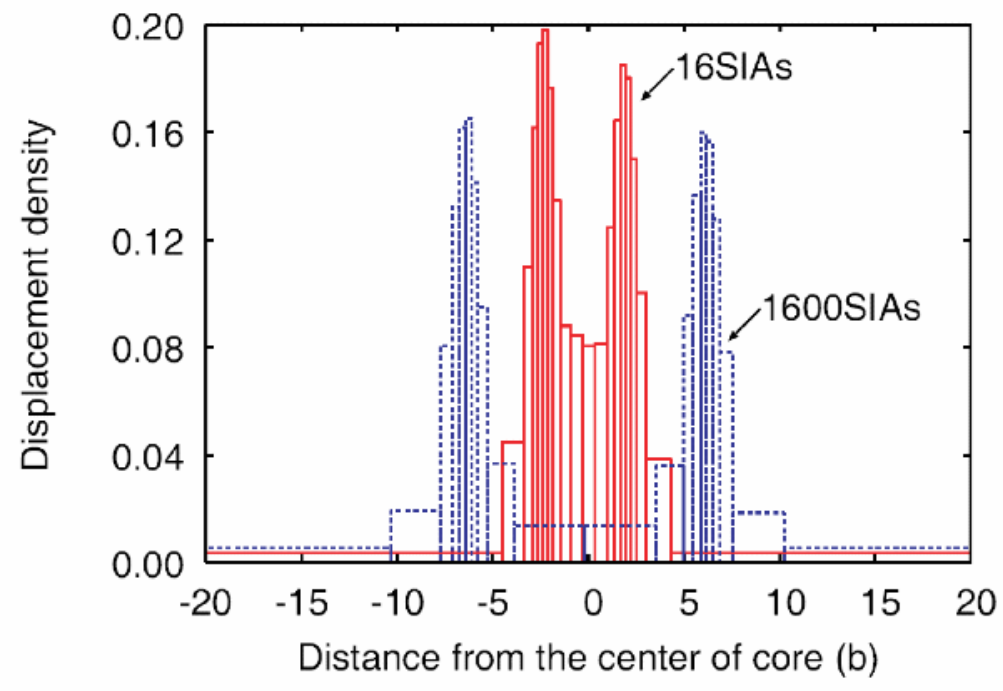

Figure (11) 


\section{[3.3] Dislocation Dynamics Modeling}

The objective of this part of our research is to investigate fundamental aspects of dynamics of dislocation interaction with radiation-induced defect clusters, specifically with both glissile and sessile SIA clusters in defect clouds formed along grown-in dislocations. We developed KMC simulations that describe the formation of SIA cluster clouds during neutron irradiation in bcc Fe and their evolution kinetics. The main thrust of these simulations is to determine the size and space distribution of SIA clusters in the cloud region at a variety of displacement damage doses, taking both edge and screw dislocations into account. These results are then used as input to Parametric Dislocation Dynamics (PDD) simulations to determine the mechanisms and the critical stresses of dislocation unlocking from defect atmospheres. The motion and interaction of SIA clusters can lead to the formation of rafts of small dislocation loops and decoration of dislocations. The multiplication and movement of these free dislocations and the resulting dislocation-dislocation interactions leads to work hardening as plastic deformation continues.

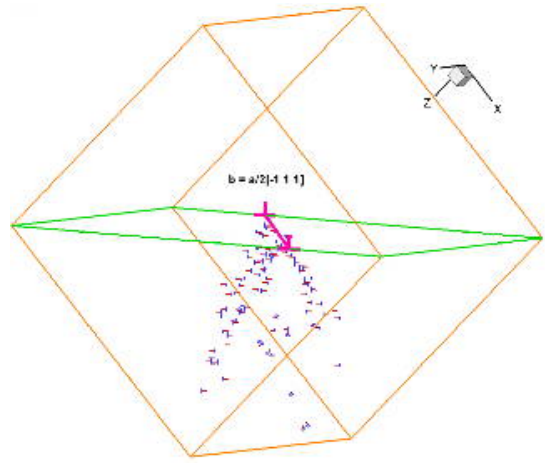

(a)

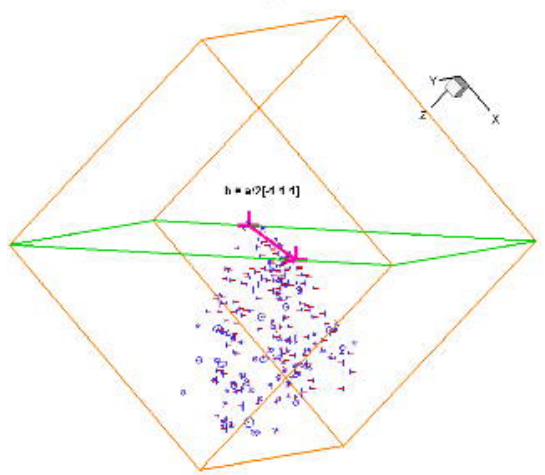

(c)

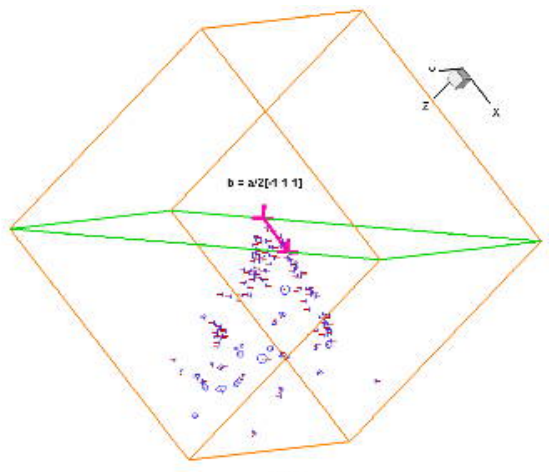

(b)

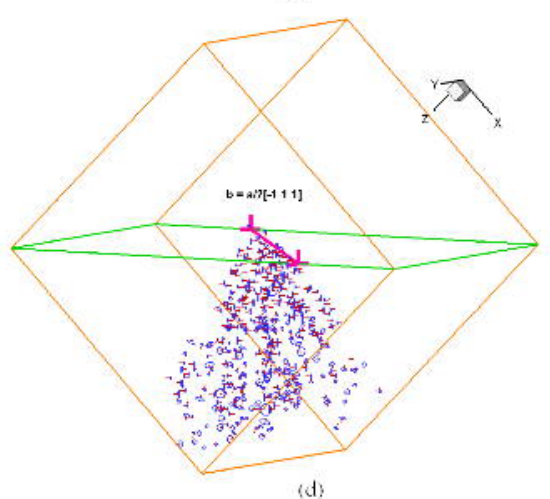

(d)

Figure (13): KMC simulation results of SIA cluster cloud evolution on the tensile side of an edge dislocation in bcc $\mathrm{Fe}$ irradiated at $300 \mathrm{~K}$ to a displacement dose level of (a) $2 \times 10^{-4}$ dpa, (b) $4 \times 10^{-4} \mathrm{dpa}$, (c) $0.001 \mathrm{dpa}$, and (d) $0.002 \mathrm{dpa}$, respectively. The edge dislocation has a Burgers vector $\mathrm{b}=$ 
We first consider the models that involve the interaction between an edge dislocation and a number of SIA clusters. A computational cell of $300 \mathrm{a} \times 300 \mathrm{a} \times 300 \mathrm{a}(\mathrm{a}=0.2867 \mathrm{~nm}$ is the lattice constant of iron) is used with periodic boundary conditions. An edge dislocation with $\mathbf{b}=\mathrm{a} / 2[-$ 111] lying on a (101) plane is inserted in the simulation box. We performed KMC simulations for microstructure evolution in pure iron irradiated at $300 \mathrm{~K}$ to displacement dose in the range of $2 \times 10^{-4}-2 \times 10^{-3} \mathrm{dpa}$. A fixed initial number of SIA clusters with a size distribution obtained from MD simulations, and corresponding to damage dose levels of $2 \times 10^{-4}, 4 \times 10^{-4}, 0.001$, and 0.01 $\mathrm{dpa}$, respectively, were introduced randomly into the simulation cell. To give a clear view of the structure of the cloud, we excluded the freely moving SIA clusters and only those that were trapped in the cloud region are shown in Figure 13. Even at low damage doses, for example at 0.0002 dpa as shown in Figure 13-a, SIA clusters are already trapped in the region of positive dilatational strain in the vicinity of the edge dislocation, where the elastic interaction between the dislocation and the SIAs is attractive. The clusters formed two wings that are $30 \pm$ with respect to the normal direction of the slip plane of the edge dislocation. It is observed that most of the trapped defect clusters have Burgers vectors that are parallel to that of the edge dislocation. In view of the initial random distribution of SIA clusters' Burgers vectors, the present results confirm that SIA clusters near dislocations rotate their Burgers vectors in response to the influence of the strain field of the edge dislocation. As the displacement damage dose level increases, the two wings consisting of SIA clusters continue to grow, and become more packed and prominent because of additional cluster trapping. With further agglomeration of SIA clusters in extended cluster wings, trapped clusters start to precipitate in between the two wings and lead to the eventual disappearance of the distinct and separate wings, as shown in Figure 13-c. Figure 13-d shows that at $0.002 \mathrm{dpa}$, a wedge-shaped SIA cluster \cloud" has been fully developed along the dislocation line.

We also investigated the dislocation behavior locally decorated by a SIA cloud, and evaluated the critical shear stress. In the simulation, the relative positions of the dislocation and the SIA cloud are absolutely the same as the result of the KMC simulation. Figure 14 shows an example of the dislocation behavior interacting with the SIA cloud formed $2 \times 10^{-3} \mathrm{dpa}$. In the Figure, the middle of the dislocation, which is decorated by SIA clusters, is strongly affected by the SIA cloud. Then only the part of the dislocation cannot move while the rest part of the dislocation already starts to glide on the slip plane. The behavior shows us that the effect of the SIA cloud on the dislocation is clearly a pinning effect. The bow out of the dislocation is extended by the increase of the external shear stress. The dislocation can break away from the SIA cloud when the external shear stress gets sufficient to break away from the SIA cloud. 


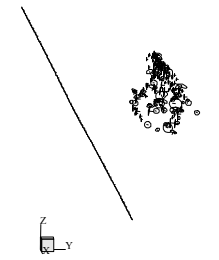

(a) (

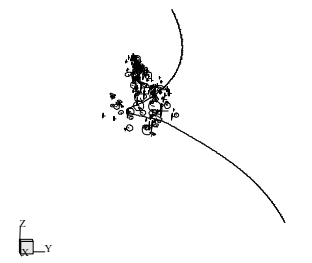

(c)

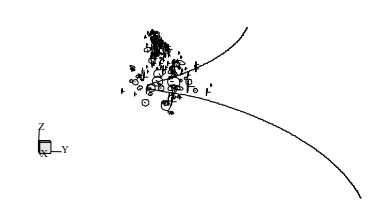

(e)

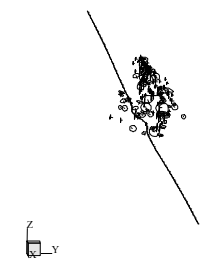

b)

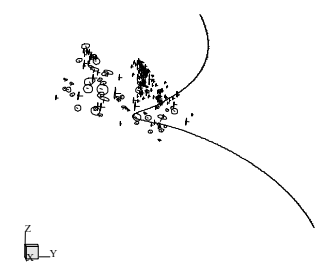

(d)

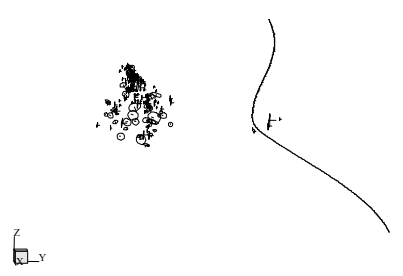

(f)

Figure 14: Dislocation behavior during breaking away from a decoration by an SIA cloud. The SIA cloud clearly works as an obstacle which gives a pinning effect to the dislocation. The applied stress (in MPa) at each snapshot is: (a) 12; (b) 17;(c) 22; (d) 27: (e) 32:(f) 


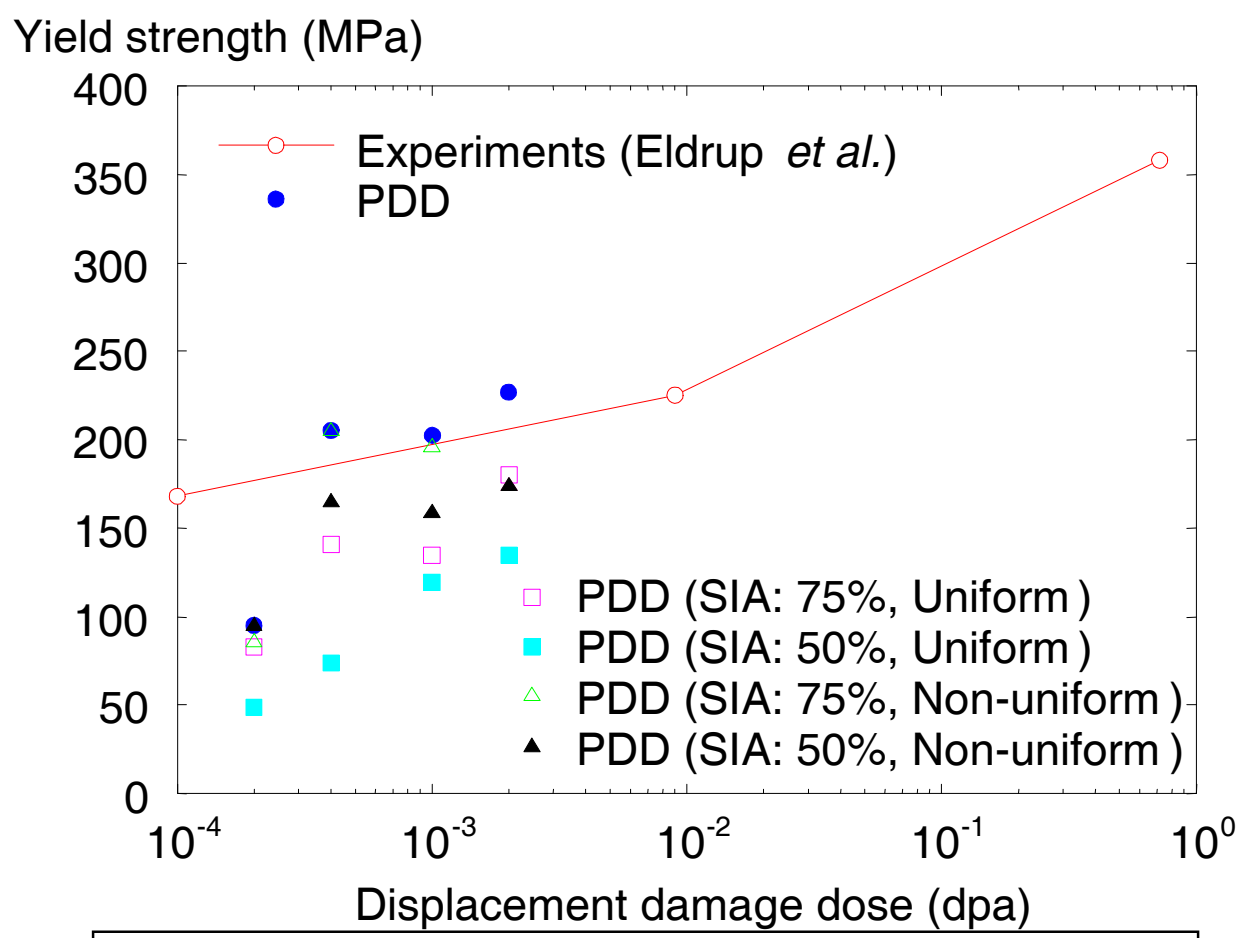

Figure 15: Yield strength as a function of displacement damage dose. PDD simulation results are plotted with solid circles, and experimental results obtained by Eldrup et al. are plotted as circles with connecting line. PDD simulation results with different recombination fractions are also shown. The results with SIA clouds, which are uniformly reduced by $25 \%$ and $50 \%$ of SIA clusters, are respectively shown with hollow and solid squares. The results with SIA clouds, which are nonuniformly reduced by $25 \%$ and $50 \%$ of SIA clusters, are plotted with hollow and solid triangles, respectively.

Eldrup et al: performed irradiation experiments on Fe in the High Flux Isotope Reactor (HFIR), and determined the stress-strain relationships in tension at a strain rate of $1.2 \times 10^{-3} \mathrm{~s}^{-1}$. Using a Taylor factor of 3.06, we show in Figure 15 a comparison between their experiments and results of the present simulations. Computer simulations are performed for source hardening alone without accounting for work-hardening by dislocation-dislocation interactions. In the model used here, SIA clusters corresponding to a particular dose are introduced suddenly in the simulation 
volume and are allowed to diffuse one-dimensionally till they self-trap or they aggregate around the dislocation in a cloud. Thus, one expects that the gradual build-up of SIA clouds in the experimental situation should result in weaker interactions because of recombination events that take place over time. Notwithstanding these limitations, the comparison between experiments and the current model should be taken as qualitative at best, and with these limitations borne in mind. At the low dose of $2 \times 10^{-4} \mathrm{dpa}$, the measured value of radiation hardening (i.e. increase in yield stress) is significantly lower than the experimental value indicating that the major contribution to the yield strength in the experiments may be the dislocation-dislocation interactions, and not the source hardening effects of SIA clusters. On the other hand, the calculated values of the increase in the equivalent uniaxial tensile stress at $4 \times 10^{-4}, 10^{-3}$ and 2 $\times 10^{-3} \mathrm{dpa}$ are in reasonable agreement with the experimental results.

\section{[3.4.] Constitutive \& Crystal Plasticity Modeling}

The objective of this research is to develop an understanding of the mechanical behavior and dislocation microstructure evolution of single and polycrystals, and to delineate the physical and

mechanical origins of spatially-localized plastic deformation. Traditional approaches to the study of plastic instabilities have either been based on kinematics considerations, such as finite strain effects and geometric softening, or physics-based concepts. In this study, we developed a framework that combines both approaches. A rate-independent crystal plasticity model was developed to incorporate micromechanics, crystal structure and microstructure into a continuum description of finite strain plasticity. A comprehensive dislocation density model based on rate theory is employed to determine the strain hardening behavior within each plastic slip system for the fcc crystal structure. Finite strain effects and the kinematics of crystal plasticity are coupled with the dislocation-density based model via the hardening matrix in crystal plasticity. ABAQUS/CAE is employed as a finite element method solver, and several user's subroutines were developed to model fcc crystals with 2 and 12 slip systems. The developed material models are applied to study single and polycrystal deformation behavior of copper. Interfaces between the ABAQUS user's subroutine Umat and the ABAQUS main code are developed to allow further extension of the current method. Simulations carried out for polycrystals clearly illustrate the heterogeneous nature of plastic strain, and the corresponding spatial heterogeneity of the mobile dislocation density. The origins of the spatial heterogeneities are essentially geometric, as a result of constraints on grain rotation (finite strain effects), geometric softening due to plastic unloading of neighboring crystals. The physical origins of plastic instabilities manifest themselves in the coupling between the dislocation densities and the localized kinematically induced softening. Representative results are shown in figure 16. 


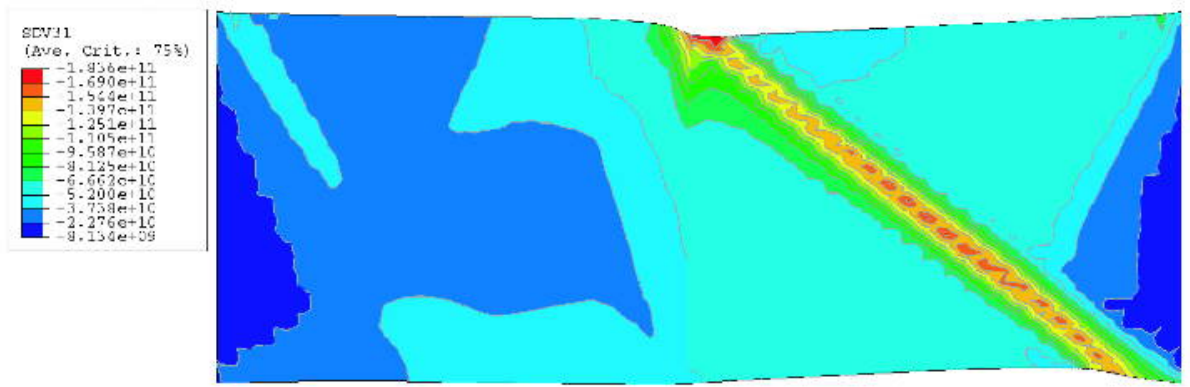

(a)

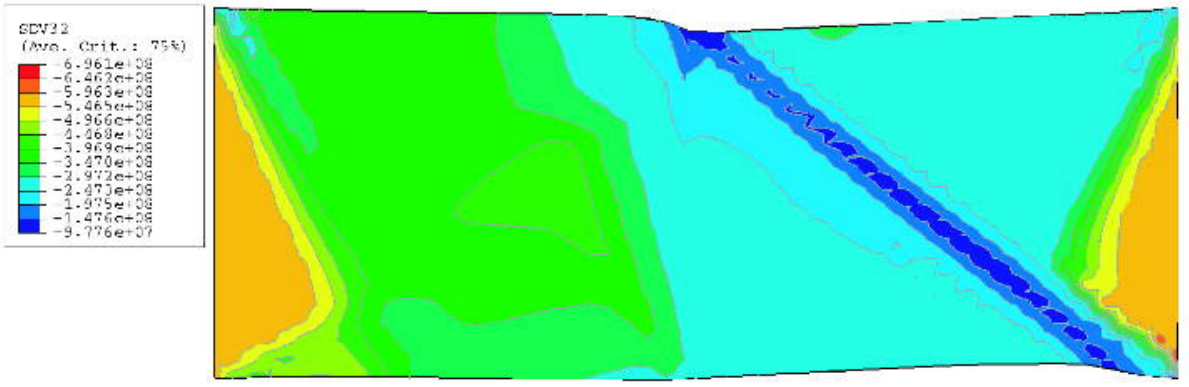

(b)

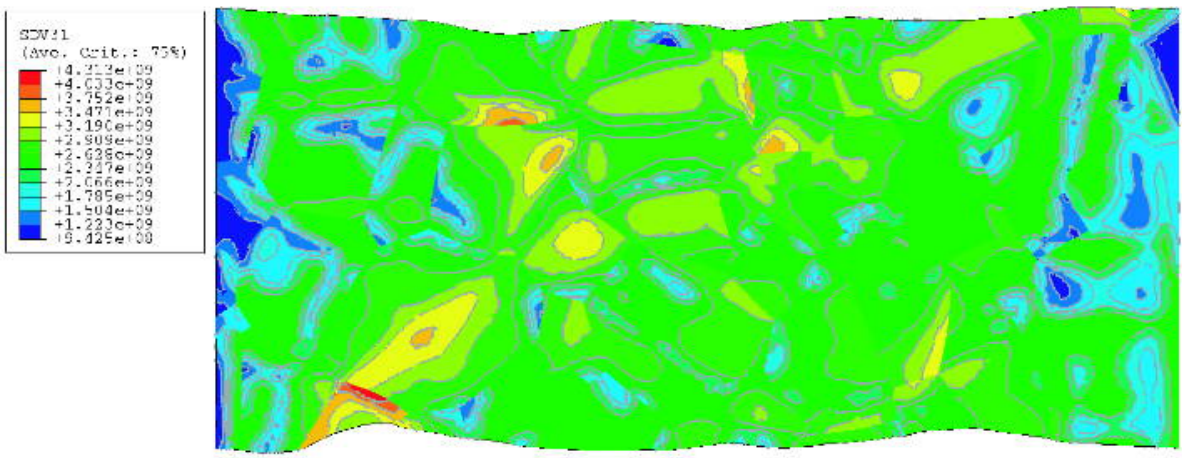

Figure 16: Distribution of plastic strain and dislocation density in an Fe bicrystal. 


\section{[4] References}

1. G. Wang, A. Strachan, Tahir Çağin, and William A. Goddard III, Phys. Rev. B 67, 140101(R) (2003).

2. M.S. Duesbery and V. Vitek, Acta Mater. 46, 1481 (1998).

3. H. Suzuki, in Fundamental Aspects of Dislocation Theory, edited by J.A. Simmons, R. deWitt and R.Bullough ( Washington, National Bureau of Standards), p.253.

4. S.L. Frederiksen and K.W. Jacobsen, Philos. Mag. A 83, 365 (2003).

5. C. Domain and G. Monnet, Phys. Rev. Lett. 95, 215506 (2005).

6. E. Pink and R. J. Arsenault, Prog. Mater. Sci. 24, 1 (1979).

7. W.H. Gourdin, D.H. Lassila, M.M. LeBlanc, and A.L. Shields, J. de Physique IV, Colloque C8, 207 (1994).

8. C.L. Briant, D.H. Lassila, J. Eng. Mater. Technol.-Trans. ASME 121, 172 (1999).

9. C. Woodward, and S.I. Rao, Phys. Rev. Lett. 88, 216402 (2002).

10. N. Choly, G. Lu, Weinan E, and E. Kaxiras, Phys. Rev. B 71, 094101 (2005).

11. G. Wang, A. Strachan, Tahir Çağin, and William A. Goddard III, Mater. Sci. Eng. A 309, 133 (2001).

12. L.H. Yang, P. Söderlind, and J.A. Moriarty, Philos. Mag. A 81, 1355 (2001).

13. S. Lozano-Perez, M. L. Jenkins, and J. M. Titchmarsh, Philos. Mag. Lett. 86, 367 (2006).

14. K. Nogiwa, T. Yamamoto, K. Fukumoto, H. Matsui, Y. Nagai, K. Yubuta and M. Hasegawa, J. Nucl. Mater. 307-311, 946 (2002); K. Nogiwa, N. Nita and H. Matsui, J. Nucl. Mater. 367-370, 392 (2007).

15. K. Nogiwa, N. Nita and H. Matsui, J. Nucl. Mater. 367- 370, 392 (2007).

16. R.J. Gaboriaud and M. Boisson, J. de Physique, Colloque C6, 171 (1980).

17. R.J. Gaboriaud, P. Veyssière J. Rabier and M. Boisson, J. Mater. Sci. 13, 907 (1978). 